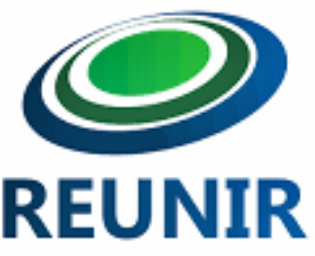

REUNIR:

Revista de Administração, Ciências Contábeis e Sustentabilidade

ARTIGO ORIGINAL

\title{
Gerenciamento de Resultados e Avaliação de Empresas: Uma Análise da Relevância dos Accruals Discricionários no Modelo de Ohlson ${ }^{1}$
}

\section{Earnings Management and Business Valuation: An Analysis of the Relevance of Discretionary Accruals in the Ohlson Model}

\section{Gestión de Resultados y Evaluación de Empresas: Un Análisis de la Relevancia de los Accruals Discrecionales en el Modelo de Ohlson}

\section{Kallyse Priscila Soares de Oliveira², Paulo Roberto Nóbrega Cavalcante ${ }^{3}$}

\section{PALAVRAS-CHAVE}

Gerenciamento de Resultados; Avaliação de Empresas; Value Relevance.

\section{KEYWORDS}

Earnings Management. Valuation. Value Relevance.

\begin{abstract}
Resumo:
O objetivo desta pesquisa consistiu em verificar o reflexo do gerenciamento de resultados (accruals discricionários) sobre a avaliação de empresas brasileiras de capital aberto no período de 2012 a 2017. Foram analisadas 1.170 observações, com uma média de 200 empresas por ano. Realizou-se a estimação do gerenciamento de resultados (GR) pelo modelo de Dechow et al. (2012). Em seguida, analisou-se o value relevance do gerenciamento de resultados pelo modelo de Ohlson (1995). Os principais achados confirmaram que há heterocedasticidade entre os dados da amostra, justificando o uso de regressões quantílicas. Em achados adicionais a outras pesquisas, com relação ao aumento do value relevance do modelo de Ohlson (1995), o GR apresentou relevância entre as empresas de médios e maiores valor de mercado, indicando que o gerenciamento de resultados afeta a precificação dessas firmas.
\end{abstract}

\begin{abstract}
:
The aim of this paper consisted to check the reflection of earnings management (discretionary accruals) on the of evaluation of public brazilian companies, in the period from 2012 to 2017. We analyzed 1,170 observations, with an average of 200 companies per year. The estimation of the earnings management (EM) was performed by Dechow et al. (2012). The value relevance of earnings management was then analyzed by the Ohlson model (1995). The main findings confirmed that there is heterogeneous between the sample data, justifying the use of quantile regression. In additional findings to other research, with regard to the increase of the value relevance of the Ohlson model (1995), the EM has shown importance among the companies of medium and higher market value, indicating that earings management affects the pricing of these Firms.
\end{abstract}

\section{PALABRAS CLAVE}

1 Sut
Gestión de resultados. Evaluación de empresas. Relevancia del valor. em 04.11.2018. Ajustado em 27.11.2018. Aceite em 28.12.2018. Publicado em 31.12.2018. Responsável Universidade 'ROFIAP / CCJS / UFCG. DOI 10.18696/reunir.v8i2.826

sora da UFERSA, Mossoró, RN, Brasil, e-mail: kallysepriscila@hotmail.com, ORCID: https://orcid.org/0000-0003-1693-0109. jilidade - USP, Professor PPGCC - UFPB, João Pessoa, PB, Brasil, e-mail: prncavalcante0907@gmail.com, ORCID: 
El objetivo de esta investigación se basó en verificar el efecto de la gestión de resultados (discrecionales accruals) sobre la evaluación de empresas brasileñas que cotizan en bolsa en el período de 2012 a 2017. Se analizaron 1.170 observaciones, una media de 200 empresas al año. Se realizó la estimación de la gestión de resultados (GR) por el modelo de Dechow et al (2012). A continuación, se analizó la relevancia del valor de la gestión de resultados a través del modelo de Ohlson (1995). Los principales hallazgos confirmaron que hay heterocedasticidad entre los datos de la muestra, lo que justifica el uso de regresiones cuantílicas. En los hallazgos adicionales a otras investigaciones, en relación al aumento de la relevancia del valor del modelo de Ohlson (1995), el GR presentó relevancia entre las empresas de medianos y de mayores valores de mercado, lo que indica que la gestión de resultados afecta el cálculo de precios de esas empresas.

\section{Introdução}

Entre as informações regularmente disponibilizadas pela contabilidade, para suprir a demanda daqueles que têm interesse na entidade que reporta, certamente a informação relativa ao desempenho econômico está entre aquelas que são consideradas como mais relevantes. Uma justificativa para tal visão de relevância pode ser encontrada no conjunto de decisões que são tomadas, tanto por usuários internos, quanto por usuários externos, com base no desempenho econômico.

Ao mesmo tempo que o resultado se constitui em informação relevante, a sua mensuração engloba aspectos a respeito dos quais ainda existem polêmicas, seja no meio acadêmico, seja no âmbito profissional, aspectos tais que, até pela ausência de consenso, tanto de uma definição conceitual mais robusta, quanto de critérios de mensuração, continuam a exigir o desenvolvimento constante de pesquisas (Dechow \& Dichev, 2002; Durán-Vázquez, LorenzoValdés, \& Martín-Reyna, 2012; Ohlson, 2014).

Nesse campo de discussão e de possíveis pesquisas, o tema gerenciamento de resultados ganha corpo, isto porque a utilidade dos números contábeis perderia o sentido na medida em que houver dúvida sobre a qualidade da informação reportada. A literatura contábil, nacional e internacional, tem explorado o tema gerenciamento de resultados por diversas abordagens mas, quase sempre, apresentando-o como uma das dimensões da qualidade da informação contábil, sendo capaz de produzir impacto na tomada de decisão por parte do usuário da informação (Durán-Vázquez et. al., 2012).

Ainda que do ponto de vista conceitual a ideia de gerenciamento de resultados seja de fácil compreensão, ao se considerar a mensuração dessa prática a questão assume certo nível de complexidade. Nesse sentido, é de se compreender os esforços dedicados na tentativa de aferir adequadamente e explicar os efeitos de tal prática, tarefa que encontra obstáculos de realização, no mínimo, nos aspectos: (a) relação entre fluxos de caixa, lucros evidenciados e accruals; (b) o uso de acrruals como critério de mensuração, já que tal proxy pode ter origem tanto no contexto das atividades normais da empresa, quanto no poder discricionário do gestor (Ohlson, 2014).

É importante perceber que accruals, do ponto de vista conceitual, refere-se aos valores que fazem parte do resultado apurado pela contabilidade, e que não implicam em movimentação de disponibilidades no período, objetivamente conceituado na literatura contábil como a diferença entre o lucro líquido e o fluxo de caixa do período (Dechow \& Dichev, 2002).

Deve ser notado, então, que no caso de os accruals incluídos no resultado guardarem relação com as atividades normais da empresa, deve-se ter informação de boa qualidade. Diferentemente, no caso de os accruals resultarem da discricionariedade do gestor, surge a dúvida da qualidade da informação divulgada. Em outros termos, a forma como os accruals são reconhecidos e tratados nas demonstrações contábeis afetará a qualidade dos lucros (Martinez, 2008).

Sendo a informação o produto da contabilidade, entende-se que o lucro evidenciado tem qualidade quando representa de forma justa e adequada (true and fair view) o efeito dos processos fundamentais da empresa. Tal característica de qualidade é que concede utilidade à informação contábil para servir como suporte ao processo de tomada de decisão dos usuários. Portanto, a qualidade do lucro requer accruals vinculados às atividades normais da entidade, ou seja, não discricionários, o que implica que devem estar afastados da participação do gestor na sua definição e, não gerados por erros de estimativas (DuránVázquez et al., 2012).

Do ponto de vista do mercado de valores mobiliários, o efeito das decisões dos investidores pode ser compreendido pela variação do preço dos títulos ali negociados. Considerando a associação com fluxos de caixa como fator crítico para a tomada de decisões, convém verificar se falta de qualidade da informação contábil, provocada pela presença de accruals discricionários, tem um impacto sobre o comportamento do mercado.

Como base nas discussões até aqui colocadas, surge o seguinte questionamento da pesquisa: Qual é o reflexo do gerenciamento de resultados no processo de avaliação de empresas brasileiras de capital aberto?Este trabalho tem como objetivo verificar o reflexo do gerenciamento de resultados (accruals discricionários) sobre a avaliação de empresas brasileiras de capital aberto no período de 2012 a 2017.

Note-se a intenção de reunir duas questões: uma que tem a ver especificamente sobre "qualidade dos lucros" (gerenciamento de resultados) e outra, reflexo da primeira, que está focada na possibilidade de aquela informação poder ou não ser usada para explicar variações no preço dos títulos comercializados pelas empresas, buscando um dos principais objetivos da pesquisa nesse campo que é encontrar evidências a respeito do nível de relevância da informação contábil para as decisões de investidores do mercado de valores mobiliários (Durán-Vázquez et al., 2012).

Dada a relevância que a variável “accruals" apresenta no contexto da mensuração da qualidade do lucro, em pesquisas cujo objeto é a relação entre accruals discricionários e qualidade do lucro contábil, o tratamento dessa variável, tanto do ponto de vista conceitual, quanto no que se refere à construção de modelos econométricos, requer cuidados especiais, sob pena de o resultado da pesquisa não retratar a realidade pretendida. Entretanto, boa parte dos estudos não verifica o reflexo da heterogeneidade das firmas na estimativa da qualidade desses lucros.

Estudos internacionais questionam a forma como os dados são tratados em algumas pesquisas contábeis, podendo o método de regressão utilizado distorcer os 
resultados encontrados. Li et al. (2009) nos achados de suas pesquisas, relatam que as distribuições empíricas das principais medidas de contabilidade, possuem heterocedasticidade e não apresentam o pressuposto da normalidade, sugerindo que a utilização dos mínimos quadrados ordinários para estimação dos modelos, é inadequada por distorcer seus resultados.

Por isso, os referidos autores sugerem a utilização de regressões quantílicas. Para testar possíveis diferenças nos parâmetros entre empresas em diferentes segmentos da distribuição das variáveis analisadas (ou seja, o parâmetro de heterogeneidade), a regressão quantílica é mais apropriada porque permite examinar toda a distribuição das variáveis explicativas, em vez de se concentrar em uma única medida da tendência central da distribuição. No Brasil, devido ao tamanho do seu mercado e às características dos dados contábeis, a análise a partir do uso de regressão quantílica se torna viável, especialmente por sua incipiente exploração na área contábil.

Do exposto, espera-se que os resultados contribuam para verificar influências no value relevance da informação contábil na avaliação de empresas, sendo capaz de produzir impacto na tomada de decisão por parte do usuário da informação contábil.

\section{Elementos teóricos da pesquisa}

Esta seção aborda os principais pontos acerca do Gerenciamento de Resultados por meio dos Accruals, da Avaliação de empresas pelo modelo de Ohlson (1995) e apresenta a hipótese de pesquisa.

\section{Gerenciamento de Resultados por meio dos Accrual}

O modelo contábil baseado no regime de competência incorpora a intuição de que os efeitos econômicos das transações e eventos sejam reconhecidos, independentemente do momento da realização dos fluxos de caixa relacionados. Nesse contexto, o benefício do registro contábil dos accruals é exatamente efetuar o ajuste entre o fluxo de caixa e o regime de competência (Paulo, 2007).

Ressalta-se que o registro contábil dos accruals se, por um lado, produz o benefício do atendimento às regras contábeis, descolando o efeito econômico dos eventos do seu efeito financeiro (fluxo de caixa), por outro lado, impõe o custo de se fazer suposições e estimativas as quais podem requerer correções posteriores em accruals futuros (Dechow \& Dichev, 2002).

Alguns dos erros de estimação são ocasionados pelas características idiossincráticas das firmas e pela complexidade das transações e eventos econômicos, entretanto, a magnitude dos erros de estimativas, intencionais ou não, prejudica a qualidade dos accruals incluídos no resultado $\mathrm{e}$, consequentemente, reduz a utilidade da informação contábil para os usuários (Paulo, 2007). Segundo Dechow e Dichev (2002), os resíduos da regressão dos fluxos de caixa presentes, passados e futuros são os accruals que não estão vinculados com a realização do fluxo de caixa, e o desvio-padrão ou o valor absoluto desses resíduos é uma medida de qualidade dos accruals.

Neste sentido, pretendendo-se utilizar os accruals discricionários como proxy para gerenciamento de resultados, entende-se que quanto menores os valores desses accruals, menor é a ocorrência de erros de estimativas ou manipulação de resultados por parte do gestor, o que implica em um lucro de maior qualidade.
No processo de geração de valor da empresa, os lucros divulgados podem exercer influência no custo de capital neste caso ganha relevância a questão da estimação e do reconhecimentos dos accruals -, em virtude da relação com a qualidade dos lucros. Assim, existem evidências da relação entre o custo de capital próprio e da qualidade dos lucros por meio da qualidade dos accruals, com base no retorno das ações realizadas, indicando que a qualidade dos accruals está positivamente correlacionada com retornos realizados em ações individuais (Ogneva, 2012)

A qualidade dos accruals é um tema explorado de diversas maneiras na literatura contábil internacional e nacional (Cupertino \& Martinez, 2008; Formigoni, Antunes, Paulo, \& Pereira, 2012; Joia \& Nakao, 2014, Dutra \& Costa, 2014; Ogneva, 2012). Os pontos mais analisados giram em torno da qualidade da informação contábil, mais presente na dimensão do gerenciamento de resultado. Neste sentido, seguem abaixo os principais pontos de pesquisas que abordam o tema.

Cupertino e Martinez (2008) proporam um procedimento de revisão analítica para seleção de empresas para auditoria, tendo por base o nível dos accruals discricionários presentes nas demonstrações financeiras dos anos de 1995 a 2005. O estudo concluiu que os níveis dos accruals servem como uma medida elementar da manipulação dos resultados contábeis, que deve ser corroborada com outros elementos disponíveis, servindo como indício para orientar potenciais auditorias.

Formigoni et al.(2012)verificaram se o gerenciamento de resultados contábeis, por meio dos accruals, é incentivado por questõestributárias no período de 2000 a 2005. Os resultados apontaram que os modelos econométricos têm baixo poder preditivo, não se podendo afirmar queos incentivos tributários afetam a mensuração dos componentes contábeis patrimoniais e deresultado de companhias abertas brasileiras.

Joia e Nakao (2014) verificaram se houve mudança nos níveis de gerenciamento de resultados após 2010 e se foi em função da adoção completa de IFRS pelas companhias brasileiras de capital aberto (com exceção das instituições financeiras) no período de 2006 a 2011. Os resultados não confirmaram a hipótese de que a adoção delFRS afetou o nível de gerenciamento de resultados, através dos accruals discricionários no período analisado, mas mostraram que o tamanho e o endividamento explicam significativamente os accruals discricionários, independentemente da adoção de IFRS.

Dutra e Costa (2014)investigaram se existe relação entre 0 gerenciamento de resultados e 0 grau de conservadorismo de companhias abertas brasileiras. A primeira etapa da metodologia consistiu no cálculo dos accruals discricionários. Em seguida, foram rodados modelos de regressão para a identificação do conservadorismo contábil para o resultado divulgado e para o resultado que contempla somente os accruals não discricionários, proxy para o "resultado não gerenciado". Os resultados indicaram que não existe diferença entre o grau de conservadorismo do resultado divulgado em comparação ao resultado não gerenciado.

\section{Avaliação de Empresas: Modelo de Ohlson (1995)}

Os modelos de value relevance, entre eles o modelo de Ohlson (1995), são importantes para verificar se os números contábeis são significativos para a tomada de decisão dos investidores. Na ausência de significância, há que se considerar falha no processo de regulação do exercício 
contábil, já que não logrou eficácia para trazer utilidade da informação contábil aos usuários e, também, custos dos processos de emissão de normas pelos órgãos e de elaboração dos relatórios financeiros pelas empresas, que são elevados (Duarte, Girão, \& Paulo, 2017).

O modelo de Ohlson (1995) é um método de avaliação de empresas com o maior número de trabalhos de pesquisa na literatura internacional de contabilidade, referência para a análise de mercado em pesquisas na área de finanças, uma vez que a informação financeira é considerada e identificada como um componente de valor que permite visualizar a possível relação entre a relevância das informações financeiras do exercício com a resposta do mercado de ações (Durán-Vázquez et al., 2012).

Muitos são os modelos de avaliação de empresas que buscam estimar o valor das companhias, entretanto o modelo de Ohlson (1995) concerne especialmente ao value relevance dos números contábeis, além de se basear em dividendos, em valor presente dos fluxos de caixa futuros e em múltiplos de mercado (Girão, Martins, \& Paulo, 2014). Este métodoé suportado por três premissas básicas: (i) valor presente dos dividendos esperados (present value of expected dividends - PVED); (ii) lucro limpo (clean surplus relation - CSR); e (iii) um modelo linear que define o comportamento estocástico dos lucros residuais futuros.

Neste sentido, Ohlson (1995) propõe modelo linear que define o comportamento estocástico dos lucros residuais futuros, como descrito nas equações 1 e 2 .

$$
\begin{aligned}
& x_{t+1}^{a}=\omega 11 x_{t}^{a}+v_{t}+\varepsilon_{1 t+1} \\
& v_{t+1}=\gamma v_{t}+\varepsilon_{2 t+1}
\end{aligned}
$$

Nesse modelo, $x_{t}^{a}$ é o lucro anormaldo período $t$. $\left(x_{t}^{a} \equiv x_{t}-r b_{t-1}\right) ; v_{t}$ são outras informações.w11éé o parâmetro de persistência dos lucros anormais $x_{t}^{a} .(0 \leq \omega<1)$; $\gamma$ é o parâmetro de persistência de outras informações $\mathrm{U}_{\mathrm{t}}$ $(0 \leq \gamma<1)$; e $\varepsilon_{1 \mathrm{t}}, \varepsilon_{2 \mathrm{t}} \mathrm{são}$ os termos de erros. Dessa forma, a função de avaliação de uma empresa pode ser obtido conforme a equação e.

$$
V_{t}=b_{t}+\alpha_{1} x_{t}^{a}+\beta_{1} v_{t}
$$

Onde:

$$
\begin{aligned}
& \alpha_{1}=\frac{\omega 11}{1+r-\omega 11} \\
& \beta_{1}=\frac{1+r}{(1+r-\omega 11)(1+r-\gamma)}
\end{aligned}
$$

Assim o modelo linear de Ohlson (1995) é um modelo de dinâmica da informação que descreve o mecanismo do poder de lucros excessivos de uma empresa. O poder de lucros excessivos de uma empresa é uma capacidade da empresa de gerar mais lucros do que os lucros normais da empresa. Lucros anormais, portanto, podem ser interpretado como poder de lucros excessivos de uma empresa (Ohlson, 1995).

O estudo de Durán-Vázquez et al.(2012)aplicou o modelo Jones modificado (1991) para empresas selecionadas do México, com o objetivo de avaliar o impacto dos accruals discricionários nos relatórios financeiros, a fim de identificar o value relevance por meio desta variável, incluindo-a no modelo de Ohlson (1995). Os autores encontraram significância estatística nas variáveis do modelo de Ohlson (1995), entretanto, ao analisar a inclusão da variável accruals discricionários, não encontraram relevância estatística, inferindo que esta variável não afeta os preços das ações.

O value relevance indica a relevância e a confiabilidade que se pode atribuir aos números divulgados pela contabilidade. Por tal visão, na medida em que se registra um valor menor de accruals discricionários, é esperado um aumento do value relevance dos números divulgados.

Neste sentido, buscando comparar com os achados da pesquisa dos autoresDurán-Vázquez et al.(2012), devido às características particulares do mercado de ação brasileiro, quanto a estrutura de capital, concentração do poder de voto, proteção ao acionista minoritário, regulação, entre outros fatores, apresenta-se a seguinte hipótese de pesquisa:

Hipótese 1: O uso de accruals discricionários como proxy de gerenciamento de resultado permite mensurar de modo mais adequado o value relevance do modelo de Ohlson.

Procura-se colocar em evidência a importância do estudo da variável que representa o gerenciamento de resultados, entendendo-se que a mesma exerce impacto no fluxo de caixa e sobre o retorno de capital exigido pelos investidores, neste sentido segue-se a pesquisa focando no value relevance da variável analisada.

\section{Elementos metodológicos da pesquisa}

Esta seção descreve o método de pesquisa incluindo a composição da amostra e a definição dos modelos empíricos utilizados na pesquisa.

\section{Descrição da Amostra}

0 estudo foi dividido em duas partes. Na primeira parte analisou-se a estimação da variável gerenciamento de resultados, utilizando o modelo Dechow et al. (2012) por meio do método dos quadrados ordinários e da regressão quantílica, buscando a combinação de previsões quantílicas para aproximar a média condicional (previsão ótima pelo critério do mean-squared-error). $\mathrm{Na}$ segunda parte, analisou-se o value relevance da inclusão da variável gerenciamento de resultadosno modelo de avaliação de empresas de Ohlson (1995), pelos dois métodos de regressão.

O plano amostral consistiu em empresas listadas na B3 no período de 2012 a 2017 com dados disponíveis na base de dados Economática ${ }^{\circledR}$. Foram excluídas as empresas que não apresentavam dados suficientes em alguma variável proposta nos modelos e as empresas financeiras por apresentarem características particulares, como legislação específica por exemplo, que poderiam distorcer a análise dos dados. A amostra final da pesquisa ficou representada por 1170 observações em um painel desbalanceado, distribuídas da seguinte forma: 186 empresas em 2012, 193 empresas em 2013, 196 empresas em 2014, 188 empresas em 2015, 201 empresas em 2016 e 206 empresas em 2017.

Para as variáveis contábeis, foram utilizados dados anuais de demonstrações financeiras consolidadas. Ressaltase que apesar da análise consistir do período de 2012 a 2017, foram necessários coletar dados dos anos 2010 e 2011 para o cálculo de algumas variáveis utilizadas no modelo. Por isso, escolheu-se a utilização de dados divulgados no período pós IFRS, evitando que a inclusão de dados de anos anteriores causasse distorções nas estimativas das variáveis utilizadas. 
Incialmente, as amostras foram avaliadas pelos testes de Breusch-Pagan-Godfrey e de White, e os resultados sugeriram que os dados apresentaram heterocedasticidade, com significância estatística abaixo de $5 \%$, rejeitando-se a hipótese nula de que as variâncias dos erros não são constantes. Dessa forma, à medida que a variável dependente aumenta, as variâncias das variáveis independentes não variam simultaneamente, o que justifica a utilização da regressão quantílica.

Duarte et al.(2017) alertam que para testar a relevância da informação, objeto de estudo da presente pesquisa, os trabalhos nesta linha normalmente utilizam modelos com estimação pelo método dos quadrados ordinários, ao passo que, no surgimento de alguns problemas empíricos, realizam testes como a redução de escala ou scaling, tratamento de outliers, aplicação de winsorização dos dados e/ou correção da heterocedasticidade com estimações robustas, que pode acarretar em uma tomada de decisão equivocada ou à perda de informação pelo tratamento dos dados.

Neste sentido, a regressão quantílica é uma ferramenta útil para estudar dados heterocedásticos, além disso, os coeficientes estimados podem ser consistentes sob uma abordagem estocástica fraca, quando em comparação com os resultados de uma regressão de mínimos quadrados ordinários (Silva, Santos, Perobelli, \& Nakamura, 2016).

\section{Definição dos Modelos Empíricos}

Para mensuração do Gerenciamento de resultados, foi utilizado o modelo de Dechow et al. (2012), conforme equação 4.

$$
\begin{aligned}
& \Delta W C_{t}=\beta_{0}+\beta_{1}\left(1 / A_{t-1}\right)+\beta_{2}\left(\Delta R_{t}-\Delta C R_{t}\right)+\beta_{a}\left(P P E_{t}\right)+ \\
& \beta_{4}\left(\Delta W C_{t-1}\right)+\varepsilon_{t}
\end{aligned}
$$

\section{Onde:}

$\Delta W C_{t}$ é a variação dos accruals totais, que representa os accruals discricionários e não discricionários, do período $t$ - 1 para o período $t$, ponderada pelos ativos totais no final do período $t-1$;

$A_{t-1}$ é o ativo total no final do período $t-1$;

$\Delta R_{\mathrm{t}}$ é a variação das receitas líquidas entre os períodos $\mathrm{t} 1 \mathrm{e}$ $t$, ponderada pelos ativos totais no final do período $t-1$;

$\triangle C R_{t}$ é a variação das contas a receber de clientes entre os períodos $\mathrm{t}-1$ e $\mathrm{t}$, ponderada pelos ativos totais no final do período $t-1$;

$P P E_{t}$ é o saldo das contas do ativo imobilizado no período $t$, ponderado pelos ativos totais no final do período $\mathrm{t}-1$;

$\Delta W C_{t-1}$ é a variação dos accruals totais do período t-2 para o período $t-1$, ponderada pelos ativos totais no final do período $\mathrm{t}-2$;

$\varepsilon_{\mathrm{t}}$ é o erro da regressãono período $t$.

O que será considerado como proxy para gerenciamento de resultados são os valores do desvio padrão, com base no setor, dos resíduos absolutos da regressão 4, como propõe Dechow et al. (2012), representando os accruals discricionários (aqueles que possuem interferência do gestor). Assim, quanto mais próximo de 0 for 0 resíduo, menor serão os accruals discricionários, consequentemente ocorrerá menor gerenciamento de resultados, por outro lado, quanto mais distante de 0 for o resíduo, maior será o gerenciamento de resultados, levando-se em conta que as variáveis propostas no modelo de Dechow et al. (2012) expliquem com confiabilidade os accruals não discricionários (Martins, Paulo, \&Monte, 2016).

Para o cálculo da variação dos accruals totais, utilizase a equação 5, conforme explicam Dechow et al. (2012):

Onde:

$$
\Delta W C_{t}=\left(\Delta A C_{t}-\Delta P C_{t}-\Delta D i s p_{t}+\Delta D i v_{t}\right) /\left(A_{t-1}\right)(5)
$$

$\triangle W C$ é a variação dos accruals totais da empresa do período $\mathrm{t}$-1 para o período $\mathrm{t}$;

$\triangle A C_{t}$ é a variação do ativo corrente (circulante) da empresa no final do período $\mathrm{t}-1$ para o final do período $\mathrm{t}$;

$\Delta$ Disp. é a variação das disponibilidades da empresa no final do período $\mathrm{t}-1$ para o final do período $\mathrm{t}$;

$\triangle P C_{t}$ é a variação do passivo corrente (circulante) da empresa no final do período $\mathrm{t}-1$ para o final do período $\mathrm{t}$; $\Delta$ Divité a variação dos financiamentos e empréstimos de curto prazo da empresa no final do período t-1 para o final do período $\mathrm{t}$;

Todas as variáveis são ponderadas pelos ativos totais no início do período $\mathrm{t}\left(A_{\mathrm{t}-1}\right)$.

Como forma de mitigar as distorções que podem ser provocadas pela heterogeneidade das empresas da amostra, nas estimações dos accruals discricionários, estimou-se o modelo de Dechow et al. (2012) em cross-section para cada setor econômico, conforme sugerido por (MARTINS et al., 2016).

Para executar a construção da primeira parte da pesquisa, dividiu-se o estudo em etapas como explicado a seguir:

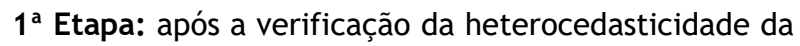
amostra, calculou-se a qualidade dos accruals, por meio da equação 4, pelo método dos quadrados ordinários.

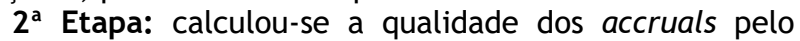
método da regressão quantílica nos quantis, $0.25,0.50$ e 0.75. Retirou-se os quantis $(0.05$ e 0.90$)$, devido as características destes apresentarem problemas de estimações, por serem considerados quantis extremos, possuindo dados mais dispersos nesta região.

$3^{\mathrm{a}}$ Etapa: combinação dos quantis $0.25,0.50$ e 0.75 , por meio de uma média aritmética dos resíduos desses quantis, buscando a combinação de previsões quantílicas para aproximar a média condicional (previsão ótima pelo critério do mean-squared-error) (Lima \& Meng, 2016).

Após essas etapas, a variável Gerenciamento de Resultados (GR) é representada pelo desvio padrão, com base no setor, dos resíduos absolutos do modelo Dechow et al. (2012), das seguintes formas:

- GR(MQO): representa os resíduos gerados pala estimação via método dos quadrados ordinários;

- GR(AV): representa a combinação dos resíduos gerados pelos quantis 25,50 e 75 da regressão quantílica, denominado Avarage (Lima \& Meng, 2016).

Para avaliar o value relevance do GR foi utilizado o modelo Residual Income Valuation (RIV) de Ohlson (1995), o qual utiliza variáveis contábeis e permite a inserção de alguma variável que possa impactar no modelo de avaliação, que o autor denomina como outras informações. Neste sentido, o modelo foi modificado, de acordo com a metodologia de Dechow et al. (2012) para a inclusão da variável Gerenciamento de Resultados, para avaliar seu impacto no valor das companhias, como explicado a nas equações 6 e 7. 


$$
\begin{aligned}
& V M_{t}=P L_{t}+\alpha_{1} L_{t}^{a}+\alpha_{2} G R(M Q O)_{t} \\
& V M_{t}=P L_{t}+\alpha_{1} L_{t}^{a}+\alpha_{2} G R(A V)_{t}
\end{aligned}
$$

Onde:

$V M_{t}$ representa o valor de mercado no período $t$, ponderado pelo ativo total do período $\mathrm{t}-1$;

$P L_{t}$ representa o patrimônio líquido no período t, ponderado pelo ativo total do período $\mathrm{t}-1$;

$L_{t}^{a}$ representa o lucro residual no período $\mathrm{t}$, ponderado pelo ativo total do período $\mathrm{t}-1$;

$G R(M Q O)_{t}$ representa o gerenciamento de resultadosno período $t$, estimado via método dos quadrados ordinários; $G R(A V)_{t}$ representa o gerenciamento de resultadosno período $t$, estimado via método regressão quantílica no quantil Avarage.

Para o cálculo do lucro residual foi necessário a multiplicação do patrimônio líquido do período anterior pela taxa de desconto livre de risco subtraindo, o lucro líquido do período atual. Este estudo utilizou como taxa de desconto livre de risco a taxa acumulada da poupança como indica Lopes (2001).

\section{Apresentação e discussão dos resultados}

O modelo Dechow et al. (2012) estima o gerenciamento de resultados por meio do desvio padrão dos resíduos absolutos do modelo da equação 4 , como explicado na seção anterior. $O$ intuito dessa metodologia é analisar qual dos dois métodos estimam menores resíduos (menores accruals discricionários), sendo assim, entende-se que o método que resultar menores resíduos por meio da equação 4, será considerado o método com menores erros de estimativas ou menores accruals discricionários, o que possibilita em menor gerenciamento de resultados por parte dos gestores, possibilitando melhor qualidade dos accruals, e consequentemente melhor qualidade dos resultados, neste caso um erro de estimativa provocado pelo método de regressão utilizado, possibilitaria em inferências distorcidas acerca desta variável.

O Gráfico 1 apresenta no eixo y os valores acumulados da diferença do desvio padrão dos resíduos em valores absolutos, os accruals discricionários, pelos dois métodos analisados (MQO-RQ) e no eixo $x$, como comporta-se esse resultado nas diferentes empresas analisadas no período de cada ano.

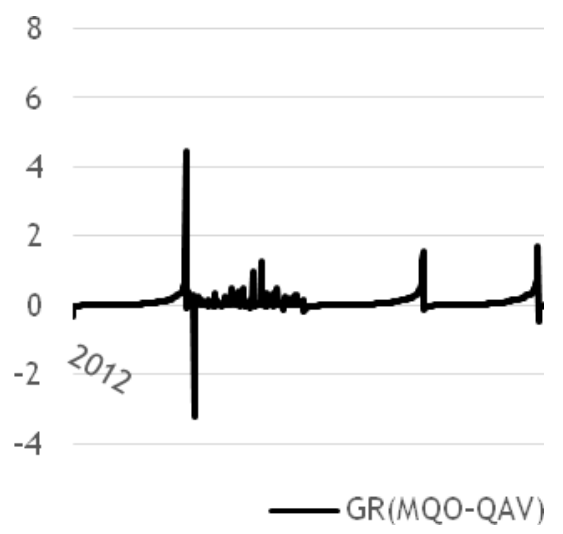

Gráfico 1: Distribuição da variável Gerenciamento de Resultados, gerada pelos resíduos da equação 6 no período de 2012 a 2017.
Fonte: Elaboração Própria (2018).

Nota: GR (MQO-QAV) (somatório acumulado da diferença das estimações do desvio padrão gerados pelo MQO e pelo Quantil Avarage).

Ao analisar o gráfico 1 percebe-se, mesmo que sutilmente, uma prevalência dos resíduos estimados por meio do método dos quadrados ordinários serem maiores que os resíduos gerados pelo método da regressão quantílica. É possível observar também a presença de outiliers na amostra, devido aos picos de valores em determinados pontos evidenciados no gráfico, ressaltando-se a existência da presença de heterocedasticidade nos dados.

Neste sentido, alerta-se que quando a variável Gerenciamento de Resultados, por meio dos accruals discricionários, estudada nesta pesquisa é analisada pelo MQO, pode-se levar uma interpretação distorcida, atribuindo o mesmo resultadopara todos os dados analisados, sem se preocupar com as diferenças contidas ao longo de toda a amostra, possibilitando informações não reais aos tomadores de decisões.

Essas observações mostram a necessidade de uma análise mais aprofundada dos dados, ressaltando que as características dos dados devem ser observadas no momento de se utilizar um método estatístico, no intuito de se utilizar a forma mais robusta, a qual possibilitará melhores inferências. Acrescenta-se que são necessárias e importantes essas observações, devido à importância do gerenciamento de resultados na literatura contábil, como discutido anteriormente.

Portanto, em seguida, incluiu-se a variável accruals discricionários, servindo de proxy para analisar o gerenciamento de resultados, no modelo de avaliação de empresas proposto por Ohlson (1995), a fim de testar o value relevance desta variável. Primeiro foi testada por meio do Método dos Quadrados Ordinários e em seguida por meio da Regressão Quantílica. Para inclusão no modelo de Ohlson (1995), foram utilizadas as variáveis GR (MQO) e GR $(A V)$, ressalta-se que a GR (AV) é a média entre os quantis.

Destaca-se que o presente estudo busca a proposta de analisar o value relevanceno sentido input-to-equityvaluation segundoHolthausen e Watts (2001), almejando tratar o papel da contabilidade como uma informação capaz de fornecer inputs para os modelos de avaliação de empresas. Sendo assim, considerou-se a estatística t para verificar se uma variável independente é significativa ou não, não testando a qualidade da utilização do modelo, mas sim somente se as variáveis independentes têm value relevance para a variável dependente, ou seja, se elas afetam significativamente ou não a variável dependente.

Como se pode perceber, de acordo com a tabela 1 , as variáveis do modelo de Ohlson (1995) se mostram significantes no mercado acionário brasileiro no período de 2012 a 2017, não importando o método de regressão utilizada, com exceção da variável lucro residual nos quantis 25 e 75, convergindo com as observações de DuránVázquez et al. (2012), os quais observam que desde 1995, o modelo de Ohlson (1995) tem sido extensivamente testado com dados dos Estados Unidos e vários países desenvolvidos e alguns países em desenvolvimento, sendo assim percebese a solidificação das variáveis que compõem o modelo. Em seus achados, Durán-Vázquez et al. (2012) encontraram value relevance no modelo de Ohlson, (1995) por meio de análise de dados em painel para todo o mercado do México no período de 2000 a 2011.

Ao analisar o impacto da variável GR (MQO), no intuito de verificar o value relevance da inclusão dessa variável no 
modelo de Ohlson (1995), no modelo 1, quando os resíduos são estimados pelo Método dos Quadrados Ordinários (MQO), a variável que representa o gerenciamento de resultados, apresenta significância estatística, sendo contrária aos achados de Durán-Vázquez et al. (2012), os quais afirmam que os accruals discricionários (proxy para gerenciamento de resultados) no mercado do México não afetam a precificação das empresas, indicando que esta variável poderia ser incorporada ao valor contábil ou lucro.

Por outro lado, quando analisado por meio da Regressão Quantílica, a variável RQ (MQO) não se mostra significante, neste caso, infere-se que quando os resíduos forem estimados via MQO, é preferível que o método que seja analisado para verificar a relação entre as variáveis seja também por MQO, entretanto esses achados trazem uma informação em média e não detalhadamente, fator este que só poderá ser visualizado no modelo 2 .

Buscando uma análise mais robusta, e no intuito de comparar os resultados obtidos, analisando as estimações do modelo de Ohlson (1995) utilizando GR (AV), como expressa o modelo 2 , os resultados para as variáveis do modelo de avaliação de empresa, são semelhantes aos resultados do modelo anterior, não importando o método de regressão utilizado, todas as variáveis se mostram significantes estatisticamente, com exceção da variável lucros residual no quantil 25, podendo-se atribuir o mesmo entendimento que ao modelo anterior.

Tabela 1 -Comparação das estimações do modelo de avaliação de empresa de (Ohlson, 1995) utilizando GR (MQO) e GR (AV).

\begin{tabular}{|c|c|c|c|c|c|c|c|c|}
\hline \multirow{2}{*}{ VARIÁVEIS } & \multicolumn{4}{|c|}{ MODELO 1: $V M_{\mathrm{t}}=P L_{\mathrm{t}}+a_{1} L_{\mathrm{t}}^{a}+a_{2} G R(M Q O)_{\mathrm{t}}$} & \multicolumn{4}{|c|}{ MODELO 2: $V M_{\mathrm{t}}=P L_{\mathrm{t}}+\alpha_{1} L_{\mathrm{t}}^{a}+\alpha_{2} G R(A V)_{\mathrm{t}}$} \\
\hline & \multicolumn{4}{|c|}{ ESTIMAÇÕES } & \multicolumn{4}{|c|}{ ESTIMAÇÕES } \\
\hline \multirow{4}{*}{$P L_{t}$} & MQO & $\begin{array}{c}\text { Quantil } \\
25\end{array}$ & $\begin{array}{c}\text { Quantil } \\
50\end{array}$ & $\begin{array}{c}\text { Quantil } \\
75\end{array}$ & MQO & $\begin{array}{c}\text { Quantil } \\
25\end{array}$ & $\begin{array}{c}\text { Quantil } \\
50\end{array}$ & $\begin{array}{c}\text { Quantil } \\
75\end{array}$ \\
\hline & $0.41^{* * *}$ & $0.28^{* * *}$ & $0.48^{* * *}$ & $0.64^{* *}$ & $0.23^{* * *}$ & $0.28^{* * *}$ & $0.63^{* * *}$ & $0.40^{*}$ \\
\hline & $(0.03)$ & $(0.08)$ & $(0.12)$ & $(0.30)$ & $(0.02)$ & $(0.05)$ & $(0.04)$ & $(0.20)$ \\
\hline & $\mathrm{t}=11.81$ & $t=3.49$ & $t=4.02$ & $\mathrm{t}=2.14$ & $\mathrm{t}=8.10$ & $t=5.15$ & $t=13.22$ & $t=1.94$ \\
\hline \multirow{2}{*}{$L^{a}{ }_{t}$} & $\begin{array}{c}-0.33^{* * *} \\
(0.03)\end{array}$ & $\begin{array}{c}-0.18 \\
(0.15)\end{array}$ & $\begin{array}{c}-0.41^{* * *} \\
(0.15)\end{array}$ & $\begin{array}{l}-0.56 \\
(0.40)\end{array}$ & $\begin{array}{c}-0.17^{* * *} \\
(0.02)\end{array}$ & $\begin{array}{l}-0.18 \\
(0.13)\end{array}$ & $\begin{array}{c}-0.53^{* * *} \\
(0.09)\end{array}$ & $\begin{array}{c}-0.35^{* *} \\
(0.15)\end{array}$ \\
\hline & $t=-10.79$ & $t=-1.16$ & $t=-2.74$ & $t=-1.39$ & $t=-6.71$ & $t=1.38$ & $t=5.47$ & $t=-2.32$ \\
\hline $\mathrm{GR}(\mathrm{MQO})_{\mathrm{t}}$ & $\begin{array}{c}0.81^{* * *} \\
(0.09) \\
t=8.42\end{array}$ & $\begin{array}{c}0.07 \\
(0.20) \\
t=0.36\end{array}$ & $\begin{array}{c}0.98 \\
(0.91) \\
t=1.08\end{array}$ & $\begin{array}{c}1.38 \\
(1.08) \\
t=1.27\end{array}$ & & & & \\
\hline $\mathrm{GR}(\mathrm{AV})_{\mathrm{t}}$ & & & & & $\begin{array}{c}1.07^{* * *} \\
(0.03) \\
t=27.48\end{array}$ & $\begin{array}{c}0.38 \\
(0.70) \\
t=0.54\end{array}$ & $\begin{array}{c}1.10^{* * *} \\
(0.10) \\
t=10.68\end{array}$ & $\begin{array}{c}1.22^{*} \\
(0.69) \\
t=1.76\end{array}$ \\
\hline Constante & $\begin{array}{c}0.74^{* * *} \\
(0.04) \\
t=18.55\end{array}$ & $\begin{array}{c}0.18^{* * *} \\
(0.04) \\
t=4.32\end{array}$ & $\begin{array}{c}0.40^{* * *} \\
(0.05) \\
t=7.69\end{array}$ & $\begin{array}{c}0.75^{* * *} \\
(0.10) \\
t=7.20\end{array}$ & $\begin{array}{c}0.68^{* * *} \\
(0.03) \\
t=21.45\end{array}$ & $\begin{array}{c}0.17^{* * *} \\
(0.03) \\
t=4.40\end{array}$ & $\begin{array}{c}0.38^{* * *} \\
(0.04) \\
t=8.46\end{array}$ & $\begin{array}{c}0.77^{* * *} \\
(0.06) \\
t=12.26\end{array}$ \\
\hline $\mathrm{R}^{2}$ & \multicolumn{4}{|l|}{0.13} & \multicolumn{2}{|l|}{0.44} & & \\
\hline F-statistic & \multicolumn{4}{|l|}{$62.85^{* * *}$} & \multicolumn{2}{|l|}{$312.65^{* * *}$} & & \\
\hline \multicolumn{9}{|c|}{ Número de observações: 1170} \\
\hline \multicolumn{9}{|c|}{ 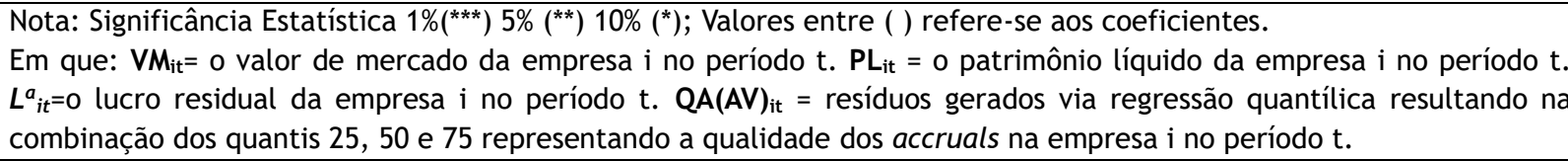 } \\
\hline
\end{tabular}

Fonte: Elaboração Própria (2017).

Ainda analisando o modelo 2 , os resultados mostram que existe diferença substancial na significância estatística quando se utiliza a combinação dos resíduos estimados via regressão quantílica, (de acordo com critérios da estatística t). Ao analisar a significância estatística da inclusão da variável GR (AV) no modelo de Ohlson (1995), verifica-se que a mesma, apresenta significância estatística a 1\%, impactando o modelo de Ohlson (1995), por meio da regressão dos quadrados ordinários, no quantil 50 também a $1 \%$, e no quantil 75 a $10 \%$, indicando que a informação contábil sobre gerenciamento de resultados traz informações significantes a respeito da precificação da empresa, principalmente nas empresas que possuem médios e altos valores de mercado.

A presente pesquisa destaca um achado adicional quando comparada às pesquisas anteriores, demonstrando que a variável gerenciamento de resultados mensurada por meio dos accruals discricionários, estimada via regressão quantílica, possui um impacto sobre o comportamento do mercado, por meio do value relevance que exerce para explicar o preço das ações das empresas, buscando um dos principais objetivos da informação financeira, a qual é encontrar evidências de que a contabilidade é relevante para as decisões de valorização dos investidores, mesmo que só encontre essa relevância, nestes dados especificamente, em empresas com médios e altos valores de mercado.

Os resultados encontrados também confirmam o estudo de Duarte et al. (2017), os quais encontraram em sua pesquisa que a regressão quantílica confere maior estabilidade intertemporal e adequação às relações teóricas dos coeficientes em comparação dos métodos dos quadrados ordinários, na maior parte dos resultados, mesmo utilizando uma outra forma da aplicação da metodologia, ressalta-se que a presente pesquisa agregou informação ao estudo, ao trazer a discussão da mensuração e inclusão da variável gerenciamento 
de resultados por meio dos accruals, diferenciando-se do estudo de (Duarte et al., 2017).

Os achados desta pesquisa também vão ao encontro com observações feitas por Girão et al. (2015) os quais observaram que o nível dos accruals discricionários das empresas é afetado significativamente pelo fluxo de caixa operacional nas empresas que reportam muitos accruals discricionários, mas não afeta aquelas que reportam poucos accruals discricionários.

Ressalta-se que só foi possível encontrar tais resultados, quando analisada a amostra mais profundamente, tendo que quando utilizado o método de regressão quantílica, o estimador deste método é derivado através da minimização de uma soma ponderada dos desvios absolutos e as estimativas dos parâmetros são menos sensíveis a outliers e caudas longas na distribuição de dados (Li et al., 2009). Isso faz com que o estimador de regressão quantílica seja relativamente robusto para heterocedasticidade dos resíduos.Outro fato é que quando a distribuição de erros se desvia da normalidade, o estimador de regressão quantílica pode ser mais eficiente do que 0 método dos quadrados ordinários (Buchinsky, 1998).

Diante do exposto, a hipótese de pesquisa pode ser verificada nas empresas que estão nos quantis 50 e 75 da amostra, indicando que a proxy para Gerenciamento de Resultados por meio dos accruals discricionários, proporciona aumento no value relevance do modelo de Ohlson (1995), apenas entre as empresas de valores de mercado em escala média e superior.

\section{Considerações Finais}

A presente pesquisa teve como objetivo geral verificar o reflexo da figura do gerenciamento de resultado (accruals discricionários) sobre a avaliação de empresas brasileiras de capital aberto no período de 2012 a 2017. Esse fator torna-se importante e necessário, devido à difícil mensuração e à diversidade de modelos para estimar o Gerenciamento de Resultados, como mostra a literatura, podendo caso apresente distorções, levar o tomador de decisão a tomar decisões com base em informações não reais no que diz respeito às empresas.

Os resultados encontrados mostram que foi verificada a presença de heterocedasticidade entre as empresas da amostra. Em seguida destaca-se que o método de regressão utilizado influencia na mensuração da variável gerenciamento de resultados, na amostra estudada entre o período analisado. No cálculo da variável gerenciamento de resultados, por meio do modelo proposto por Dechow et al.(2012), as estimações via regressão pelo método dos quadrados ordinários no geral produziram maiores resíduos que as estimadas via regressão quantílica, despertando a necessidade de uma análise mais aprofundada.

Ao analisar o value relevance do modelo de Ohlson (1995), no que diz respeito ao impacto da variável gerenciamento de resultados, na inclusão do modelo de Ohlson (1995), a significância estatística só é percebida quando avaliada via regressão quantílica, nos quantis 50 e 75, evidenciando que nos quantis inferiores, essa variável não produz impacto no processo de avaliação de empresas.
Contudo pode-se inferir que quando analisada a inclusão da variável Gerenciamento de Resultados, por meio dos accruals discricionários, no modelo de avaliação de empresa, seja estimada pelo método dos quadrados ordinários ou por meio de regressão quantílica, esta variável impacta no modelo de precificação, entretanto quando analisada por meio da regressão quantílica pode-se detalhar e ver particularidades da amostra, observando em quais grupos de empresas essa variável afeta a variável dependente, distinguindo-se de uma informação apenas pela média de tendência central.

Neste estudo, verificou-se que em média e em empresas com médios e altos valores de mercado, os accruals discricionários(proxy para gerenciamento de resultados) melhora a aproximação dos valores contábeis aos valores de mercado, possibilitando a relevância dos números contábeis, ocorrendo a melhora da qualidade da informação contábil, afetando a precificação das empresas. Neste sentido, os resultados só convergem com os de Durán-Vázquez et al. (2012), no que diz respeito à significância estatísticas nas variáveis já propostas no modelo de (Ohlson, 1995).

Acrescenta-se que só foi possível identificar resultados adicionais uma vez que se estudou a característica da amostra, testando diferenças nos parâmetros entre empresas em diferentes segmentos da distribuição das variáveis analisadas, por meio da regressão quantílica, permitindo examinar toda a distribuição das variáveis explicativas, em vez de se concentrar em uma única medida de tendência central da distribuição.

Por fim, destaca-se que os resultados aqui apresentados são restritos à amostra analisada, no período de tempo estabelecido, as quais representam apenas uma realidade simplificada, e particularidades do mercado acionário brasileiro. Entretanto, deve-se deixar claro que as limitações acima destacadas não refutam os resultados aqui encontrados, mas servem para evitar uma possível generalização dos resultados.

\section{Referências}

Buchinsky, M. (1998). Recent Advances in Quantile Regression Models: A Practical Guideline for Empirical Research. The Journal of Human Resources, 33(1), 88-126.

Cupertino, C. M., \& Martinez, A. L. A. L. (2008). Qualidade da Auditoria e earnings Management: risk assessment através do nível dos accruals discricionários. Revista Contabilidade Vista e Revista, 19(3), 69-93.

Dechow, P., \& Dichev, I. D. (2002). The Quality of Accruals and Earings: The Role of Accruals Estimation Errors. The Accounting Review, 77(2002), 35-59.

Duarte, F. C. de L., Girão, L. F. de A. P., \& Paulo, E. (2017). Avaliando Modelos Lineares de Value Relevance: Eles Captam o que Deveriam Captar? Revista de AdministraçãoContemporânea, 21(spe), 110-134.

Durán-Vázquez, R., Lorenzo-Valdés, A., \& Martín-Reyna, J. M. S. (2012). Relevance of Discretionary Accruals Information ( DAl ) in Ohlson model: the case of Mexico. Journal of Entrepreneurship, Management and Innovation (JEMI), 8(3), 21-34.

Formigoni, H., Antunes, M. T. P., Paulo, E., \& Pereira, C. A. (2012). Estudo sobre os incentivos tributários para o gerenciamento de resultados contábeis nas companhias abertas brasileiras. BASE - Revista de Administração e 
Contabilidade Da Unisinos, 9(1), 41-52.

Girão, L. F. de A. P., Martins, O. S., \& Paulo, E. (2014). Avaliação de empresas e probabilidade de negociação com informação privilegiada no mercado de capitais. Revista de Administração, 49(3), 462-475.

Holthausen, R. W., \& Watts, R. L. (2001). The relevance of value relevance literature for financial accounting standard setting. Journal of Accounting and Economics, 31, 3-75.

Joia, R. M., \& Nakao, S. H. (2014). Adoção de IFRS e Gerenciamento de Resultados nas Empresas Brasileiras de Capital Aberto. Revista de Educação e Pesquisa em Contabilidade, 8(1), 22-38.

Lima, L. R., \& Meng, F. (2016). Out-of-Sample Stock Return Prediction: What Have We Missed?. Working Paper. Forthcoming.

Martins, V. G.; Paulo, E.; Monte, P. A. (2016). O Gerenciamento de Resultados Contábeis Exerce Influência na Acurácia da Previsão de Analistas no Brasil? Revista Universo Contábil, 12(13), 73-90, 2016.

Ogneva, M. (2012). Accrual quality, realized returns, and expected returns: The importance of controlling for cash flow shocks. Accounting Review, 87(4), 14151444.

Ohlson, J. A. (1995). Earnings, books values and dividends in equity valuation.

Contemporary Accounting Research, 11(2), 661-687.

Ohlson, J. A. (2014). Accruals: An overview. China Journal of Accounting Research, 7(2), 65-80.

Paulo, E. (2007). Manipulação das informações contábeis: Uma análise teórica e empírica sobre os modelos operacionais de detecção de gerenciamento de resultados (volume I). Tese de Doutorado, 260.

Silva, E. dos S., Santos, J. F. dos, Perobelli, F. F. C., \& Nakamura, W. T. (2016). Capital Structure Of Brazil, Russia, India And China By Economic Crisis. Revista de Administração Mackenzie [online], 17(3), 105-131. 